\title{
Low Temperature Carbonization of Cellulose Nanocrystals for High Performance Carbon Anode of Sodium-Ion Batteries
}

Hongli Zhu ${ }^{1, a}$, Fei Shen ${ }^{1, a}$, Wei Luo ${ }^{1,2}$, Shuze Zhu ${ }^{2}$, Minhua Zhao ${ }^{1,3}$, Bharath Natarajan ${ }^{1,3,4}$, Jiaqi Dai ${ }^{1}$, Lihui Zhou ${ }^{1}$, Xiulei Ji ${ }^{5}$, Reza S. Yassar, ${ }^{6}$ Teng Li $^{2}$, Liangbing Hu ${ }^{1} *$

${ }^{1}$ Department of Materials Science and Engineering, University of Maryland, College Park, MD, 20742, USA

${ }^{2}$ Department of Mechanical Engineering, University of Maryland, College Park, MD 20742, USA

${ }^{3}$ Center for Nanoscale Science and Technology, National Institute of Standards and Technology, Gaithersburg, MD 20899, USA

${ }^{4}$ Materials Measurement Laboratory, National Institute of Standards and Technology, Gaithersburg, MD 20899, USA

${ }^{5}$ Department of Chemistry, Oregon State University, Corvallis, OR 97331, USA

${ }^{6}$ Department of Mechanical \& Industrial Engineering, University of Illinois at Chicago, Chicago, IL 60607, USA

${ }^{a}$ These authors contribute equally to this paper

*Corresponding author: binghu@umd.edu 


\begin{abstract}
Cellulose is the most abundant renewable material in nature. In this work, ordered cellulose nanocrystals (CNCs) have been transformed into porous carbon with an increased short-range ordered lattice and percolated carbon nanofiber at a relatively low carbonization temperature of $1000{ }^{\circ} \mathrm{C}$. When evaluated as anode for sodium-ion batteries (SIBs), the CNC derived porous carbon shows superior performances including a high reversible capacity of $340 \mathrm{mAh} / \mathrm{g}$ at a current density of $100 \mathrm{~mA} / \mathrm{g}$, which is one of the highest capacity carbon anodes for SIBs. Moreover, the rate capability and cycling stability of the porous carbon are also excellent. The excellent electrochemical performance is attributed to the larger interlayer spacing, porous structure, and high electrical conductivity arising from the ordered carbon lattice and the percolated carbon nanofiber. The formation of nano-sized graphitic carbon from the ordered $\mathrm{CNC}$ at the low carbonization temperature of $1000{ }^{\circ} \mathrm{C}$ is supported by both molecular dynamic simulations and as well as in-situ TEM measurements. This study shed light on the fundamental understanding of converting hydrocarbon biopolymer from wood to high quality carbon with a large domain of ordered lattice.
\end{abstract}

Key words: Cellulose Nanocrystals, Hexagonal Carbon, Carbon Nanofiber, Sodium-Ion Battery, Molecular Dynamic Simulation 


\section{Introduction}

Carbon is known as the most essential material for energy storage, electronics, catalysis, and other high performance multifunctional applications. ${ }^{1-8}$ Various allotropic forms of carbon including graphite, carbon nanotubes (CNT), diamond, and fullerenes et al. have been intensively studied due to their extraordinary electrical, thermal, and mechanical performances and chemical inertness. Carbon is believed to play a key role in the upcoming smart energy era for either assisting electron transfer as a conductive additive or providing ion storage as an electrode in energy storage technologies. Currently, graphite dominates the anode side in the state-of-art lithium-ion batteries (LIBs), which are the main mobile power for portable electronics and electrical vehicles. But LIBs are still too pricy for other cost-sensitive applications like grid scale energy storage. Recently, sodium-ion batteries (SIBs) have become one of the most attractive alternatives due to the abundance and low cost of sodium resources. ${ }^{9}$ Unfortunately, the limited interlayer spacing of graphite $(0.335 \mathrm{~nm})$ forbids $\mathrm{Na}$ ion intercalation, thus, hard carbon is emerging as a promising anode for SIBs due to its low cost and relatively high capacity. ${ }^{10-12}$ However, the poor rate and cycling performance of hard carbon anodes have significantly limited the overall performance of SIBs. Therefore, developing a high-performance hard carbon anode is highly desirable. ${ }^{13}$

Plants and trees are the primary carbon resources on Earth, which can convert carbon dioxide to biomass via photosynthesis. Various biomasses, such as hemp, bamboo, banana peel, have been converted to hard carbons for energy storage applications such as batteries and

supercapacitors. ${ }^{14-20}$ These precursors are typical biomass composites, which are mainly composed with cellulose, hemicellulose, and lignin with a small crystalline ratio in the range of 
$10-20 \%$. In the walls of wood fiber cells, typically approximately 36 individual cellulose molecule chains assemble with each other through hydrogen bonds and form elementary fibrils, which are further packed into larger microfibrils. The assembly of microfibrils then forms the familiar cellulose fibers. These cellulose fibers have both disordered amorphous domain and well-ordered crystalline domain. ${ }^{21,22}$ When the cellulose fibers are subjected to acid hydrolysis, the amorphous regions of fibers are selectively hydrolyzed under certain conditions because compared to crystalline domains they are more accessible to acid hydrolysis ${ }^{23}$ Consequently, the neat crystalline domains with high crystalline ratio known as cellulose nanocrystals (CNCs) are liberated from the cellulose fibers. ${ }^{24}$ Due to the highly ordered structure, the obtained CNC has been found to exhibit a theoretical Young's Modulus of 206 GPa along the [001] plane and a tensile strength of nearly $10 \mathrm{GPa}^{25,26}$

The choice of $\mathrm{CNC}$ with excellent nature-made orientation and crystallinity is important for cellulose-based carbon production due to the highly ordered structure. Regardless of the source, each cellulose chain is composed with anhydro-D-glucose via $\beta-1-4$ linkage. ${ }^{27,28}$ The wellaligned $\mathrm{CNC}$ has the right basis to form well-ordered and oriented graphitic structures. In this study, for the first time, we carbonized the neat $\mathrm{CNC}$ into porous carbon with higher graphitic structure at a relatively low temperature and used it as anode for SIBs. Well-defined hexagonal structure and long carbon nanofibers were observed within the CNC derived porous carbon. Both the experimental results and atomistic simulation support the formation of hexagonal carbon rings from glucose unit of $\mathrm{CNC}$. The electrochemical performance of $\mathrm{CNC}$ derived carbon is shown to be promising. 


\section{Experimental Section}

Preparation of CNC. Cellulose was hydrolyzed with $60 \%$ sulfuric acid at $45{ }^{\circ} \mathrm{C}$ for $6 \mathrm{~h}$. Immediately following hydrolysis, suspensions were diluted 10 -fold to stop the reaction. The suspensions were then centrifuged, washed once with water, and re-centrifuged. The resulting precipitate was placed in dialysis membranes having a molecular weight cutoff of $12000-14000$ and dialyzed against water for one week until the water $\mathrm{pH}$ remained constant.

Carbonization of CNC. The carbonized CNC electrode was obtained through thermal pyrolysis. Abovementioned $60 \mathrm{~mL}$ CNC dispersion with concentration $0.5 \mathrm{wt} \%$ was degassed $10 \mathrm{~min}$ and transferred into a $90 \mathrm{~mm}$ diameter petri dish and kept in oven at $60{ }^{\circ} \mathrm{C}$ over night. Then, the $\mathrm{CNC}$ film was heated to $240{ }^{\circ} \mathrm{C}$ with the heating rate of $1{ }^{\circ} \mathrm{C} / \mathrm{min}$ and stabilized at $240{ }^{\circ} \mathrm{C}$ for $8 \mathrm{~h}$ in air. Afterward it was further heated to $1000{ }^{\circ} \mathrm{C}$ with the heating rate of $5{ }^{\circ} \mathrm{C}$ and dwelled for $2 \mathrm{~h}$ in Argon.

Characterization. The structural (physical) analysis of the samples was evaluated by XRD using Bruker D8 Advance powder diffractometer with $\mathrm{Cu} K \alpha$ sealed tube source $(\lambda=1.5418 \AA$ ) operating at $40 \mathrm{kV}$ and $40 \mathrm{~mA}$. A JEOL 2100 microscope was employed for morphology characterization at an accelerating voltage of $150 \mathrm{kV}$. HRTEM was conducted using a FEI TITAN microscope at an accelerating voltage of $300 \mathrm{kV}$. The samples were sonicated before being transferred to the TEM grid. Zero loss Energy-filtered bright field TEM image was captured to enhance the contrast of CNC relative to amorphous carbon. In-situ TEM holder was Poseidon P-500 model from Protochips and a molybdenum grid was used for sample preparation. A Veeco MultiMode atomic force microscope with a silicon probe Tap300GD-G (Budget Sensors, USA) at a high aspect ratio tip (typical radius of curvature $10 \mathrm{~nm}$ ) was used to image the morphology of $\mathrm{CNC}$ at room temperature in an ambient atmosphere. 
Electrochemical measurements. For electrochemical testing, 2025 coin cells were made by directly punching round discs out of carbon film derived from CNC as binder-free and additivefree working electrodes. The typical mass loading of the electrode was $2 \mathrm{mg} / \mathrm{cm}^{2} .1 .0 \mathrm{M} \mathrm{NaClO}_{4}$ in $(1: 1 \mathrm{~V} / \mathrm{V})$ ethylene carbonate/diethyl carbonate served as the electrolyte, pure Na metal as the counter electrode, and a $25 \mu \mathrm{m}$ thick polypropylene membrane was used as the separator. Galvanostatic cycling was conducted with an Arbin tester at different current densities and at room temperature.

\section{Results and Discussions}

CNC has a well-ordered structure, in which the cellulose chains are tightly stacked in parallel with one another (Figure 1a). As illustrated in the zoomed-in image of cellulose molecular structure, hydrogen bonds between hydroxyl groups and oxygen of adjacent molecules enhance parallel stacking of multiple cellulose chains forming a highly ordered crystalline domain. At the early stage of carbonization, the pyrolysis of cellulose is controlled by two predominant reactions: dehydration and depolymerization. ${ }^{28}$ Dehydration is the reaction that eliminates the non-carbon elements in the biomass like hydrogen and oxygen by generating evaporable gases such as $\mathrm{CO}_{2}$, $\mathrm{CO}$, and $\mathrm{H}_{2} \mathrm{O}$. Simultaneously, the depolymerizations of the cellulose chain results in double bonds, conjugated double bonds, and even free carbon radicals, which are the participating units for the subsequent re-polymerization and atomization reactions. Using the well-ordered CNC as precursor (Figure 1b left), we obtain graphite-like layers at a carbonization temperature of 1000 ${ }^{\circ} \mathrm{C}$, which is far below the previously reported temperatures of a typical graphitization process (above $\left.1800{ }^{\circ} \mathrm{C}\right) .{ }^{29,30}$ The graphitization of carbonized $\mathrm{CNC}$ is confirmed by high-resolution transmission electron microscope (HRTEM). The left image in Figure 1b shows the long-range parallel ordered cellulose chains in the CNC. After carbonization, these chains are likely to form 
short-range ordered crystalline domains which display hexagonal arrangements of carbon atoms (Figure $1 \mathrm{~b}$ right), similar to those seen in multilayered graphene. ${ }^{31,32}$ The Fourier transform of a larger ordered region displays two sets of hexagonal reflections at $0.24 \mathrm{~nm}$ and $0.14 \mathrm{~nm}$, corresponding to the lattice constant and carbon-carbon nearest neighbor distances, respectively (see thresholded FTs shown in Figure S1). These values are in good agreement with those measured in literature, confirming the presence of conducting graphitic domains in the $\mathrm{CNC}$ derived carbon (Figure 1c). ${ }^{32-34}$ Partially graphitized $\mathrm{CNC}$ has a low resistivity of $0.13 \Omega \cdot \mathrm{cm}$, as measured by a four-probe method (see details in Supporting Information and Figure S2). 

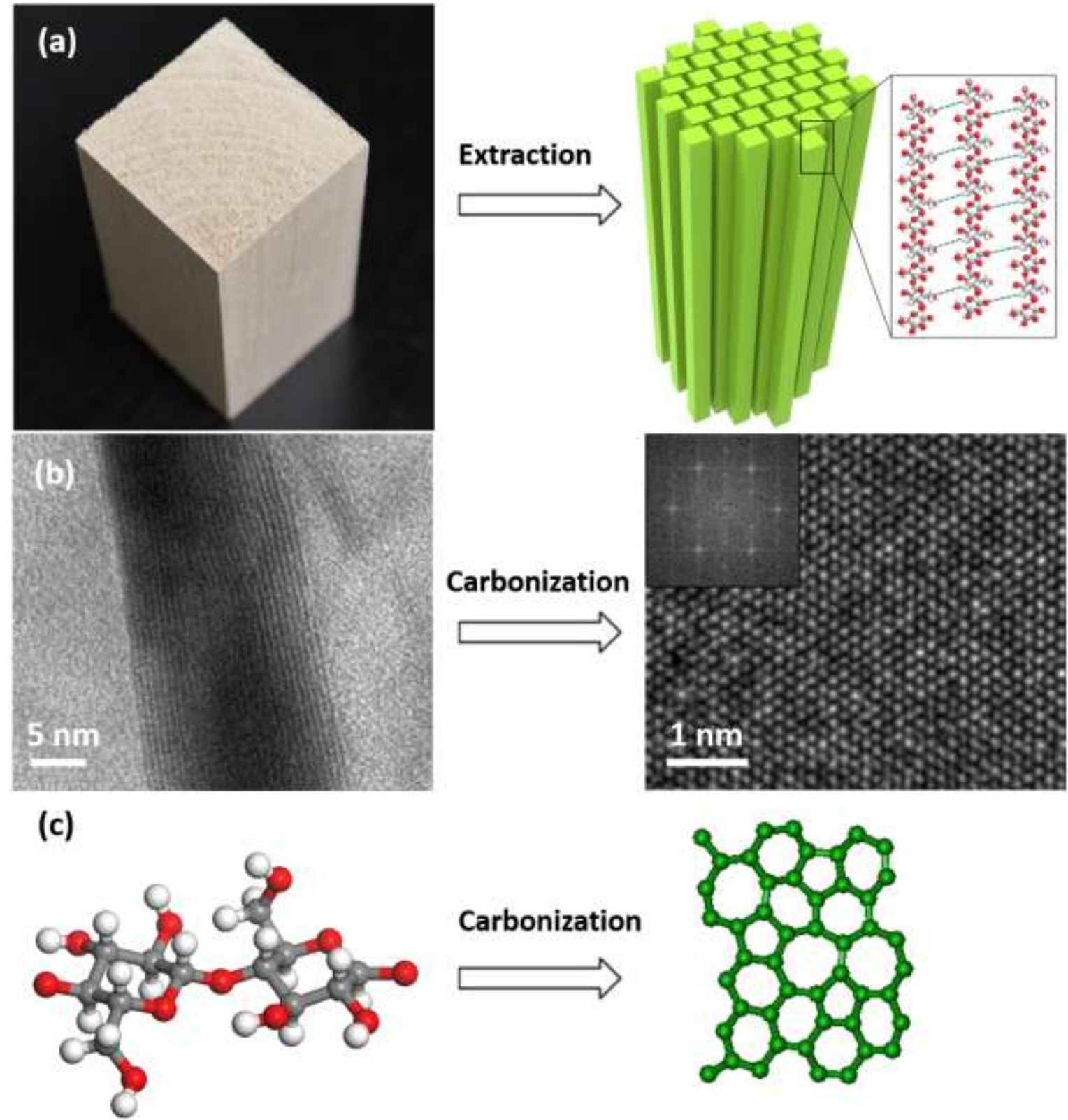

Figure 1 (a) Photograph of a wood block (left) and the schematic of CNC molecular structure extracted from wood (right); (b) HRTEM images of CNC before (left) and after carbonization (right), respectively; (c) Molecular structures of glucose unit in the cellulose chain (left) and the derived carbon ring (right). The inset in (b) showing a diffraction pattern of carbonized CNC. 
The rod-like CNC is extracted from wood, which is comprised of microfibers that have both crystalline and amorphous regions. The amorphous region is highly accessible to acid hydrolysis as shown in Figure 2a. We removed the amorphous region in regular fiber using sulfuric acid ${ }^{35-39}$, resulting in the release of the robust crystalline regions i.e. the CNC. The hydrolysis process is detailed in the experimental section. Figure $2 b$ and $2 c$ are TEM and atomic force microscope (AFM) images of CNCs cast from suspension at a dilute concentration around $5.0 \mathrm{mg} / \mathrm{mL}$, respectively. From these images, we observe that the diameter of the CNC is around 10-30 nm and the length is around $300-400 \mathrm{~nm}$. The agglomeration of CNC nanorods reflects strong intermolecular hydrogen bonding and the hydrophilic interface of the nanorods. The crystalline structure of CNC is well defined in the HRTEM image as shown in Figure 2d, in which the inset image shows the diffraction pattern. X-ray diffraction (XRD) was then used to examine the crystalline structure of regular fiber before and after hydrolysis treatment. The XRD patterns of regular fiber (Figure 2e) shows two peaks, one at $2 \theta=16.5^{\circ}$ and the other at $2 \theta=22.2^{\circ}$ assigned to (110) plane and (200) plane, respectively. ${ }^{40}$ In contrast, while the (110) and (200) planes are well maintained in $\mathrm{CNC}$, and the crystallinity is dramatically increased after acid hydrolysis. 
(a)

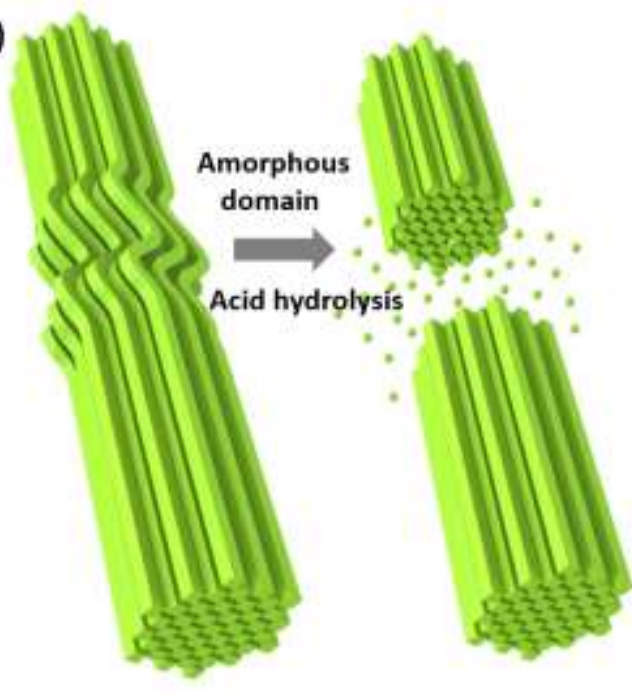

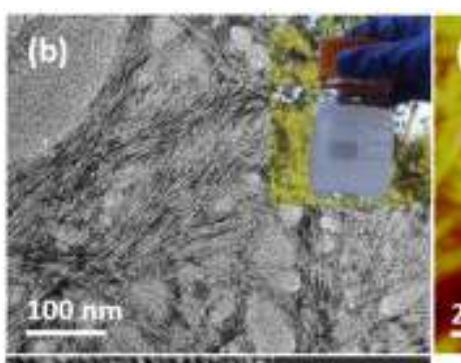
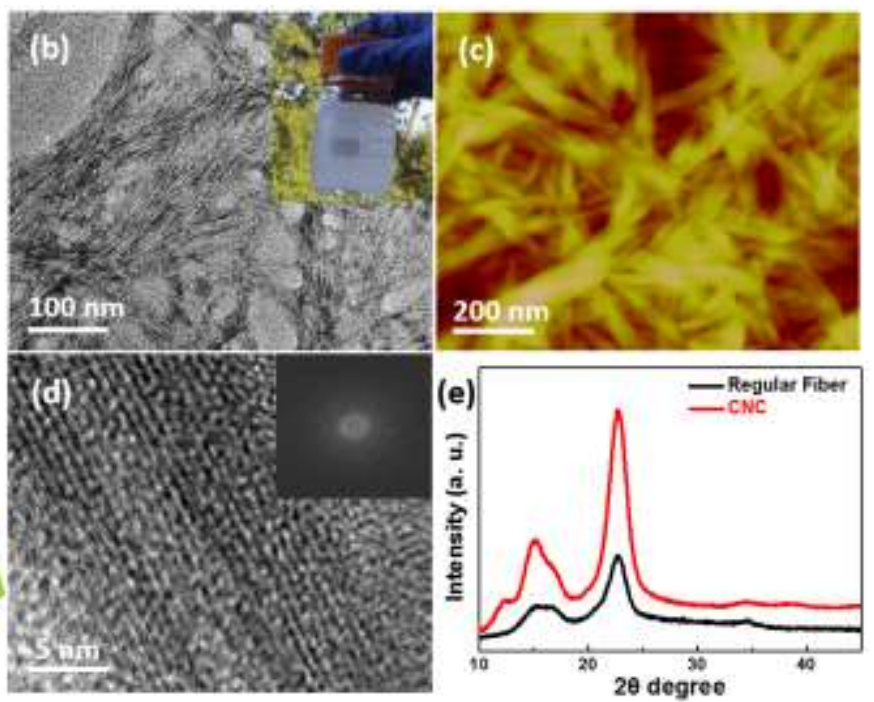

Figure 2 (a) A schematic to illustrate obtaining CNC through selective acid hydrolysis of amorphous domain in the natural wood fiber. Characterizations of CNC: (b) TEM image; (c) AFM image; (d) HRTEM image with inset showing diffraction pattern; and (e) XRD pattern. The inset of (b) shows the as-prepared CNC aqueous suspension.

Figure $3 \mathrm{a}$ and $3 \mathrm{~b}$ illustrate the transformation from $\mathrm{CNC}$ to a percolated conductive carbon by carbonization, where the red color represents carbon nanofibers. Unique mesoporous carbon with large percentages of short-range ordered lattice and a high specific ratio of carbon nanofibers was obtained from direct carbonization of $\mathrm{CNC}$ at $1000{ }^{\circ} \mathrm{C}$. In this work, we used scanning electron microscope (SEM) and TEM to examine the detailed structures with different length scales. SEM image of carbonized CNC shows a porous structure with pore diameters ranging from 20 to $50 \mathrm{~nm}$, which are uniformly distributed throughout the entire sample (Figure $3 \mathrm{c}$ ). A higher magnification SEM image (Figure 3d) shows many carbon nanofibers wrap around the mesopores, which are likely due to the one-dimensional nature of $\mathrm{CNC}$ before carbonization ${ }^{20}$. These carbon nanofibers could provide electron transfer paths, which therefore could 
dramatically improve the electrical conductivity of the carbon electrode and result in a good rate performance in batteries. HRTEM was then used to investigate the nanofibers in details. Both amorphous carbon (Figure 3e) and short-range ordered crystalline pattern (Figure 3f) are identified. Long, curved, carbon nanofiber-like structures are also found ubiquitously distributed in the porous carbon (Figure $3 \mathrm{~g}$ and $3 \mathrm{~h}$ ). The inter-layer distance in the crystalline region of short-range ordered carbon and carbon nanofibers is measured to be $0.39 \mathrm{~nm}$ and $0.32 \mathrm{~nm}$ respectively, where each layer is a $2 \mathrm{D}$ hexagonal lattice of carbon, such as in graphene. Note that the carbonization temperature is only $1000^{\circ} \mathrm{C}$. More HRTEM images of the obtained carbon are presented in Figure S3. 


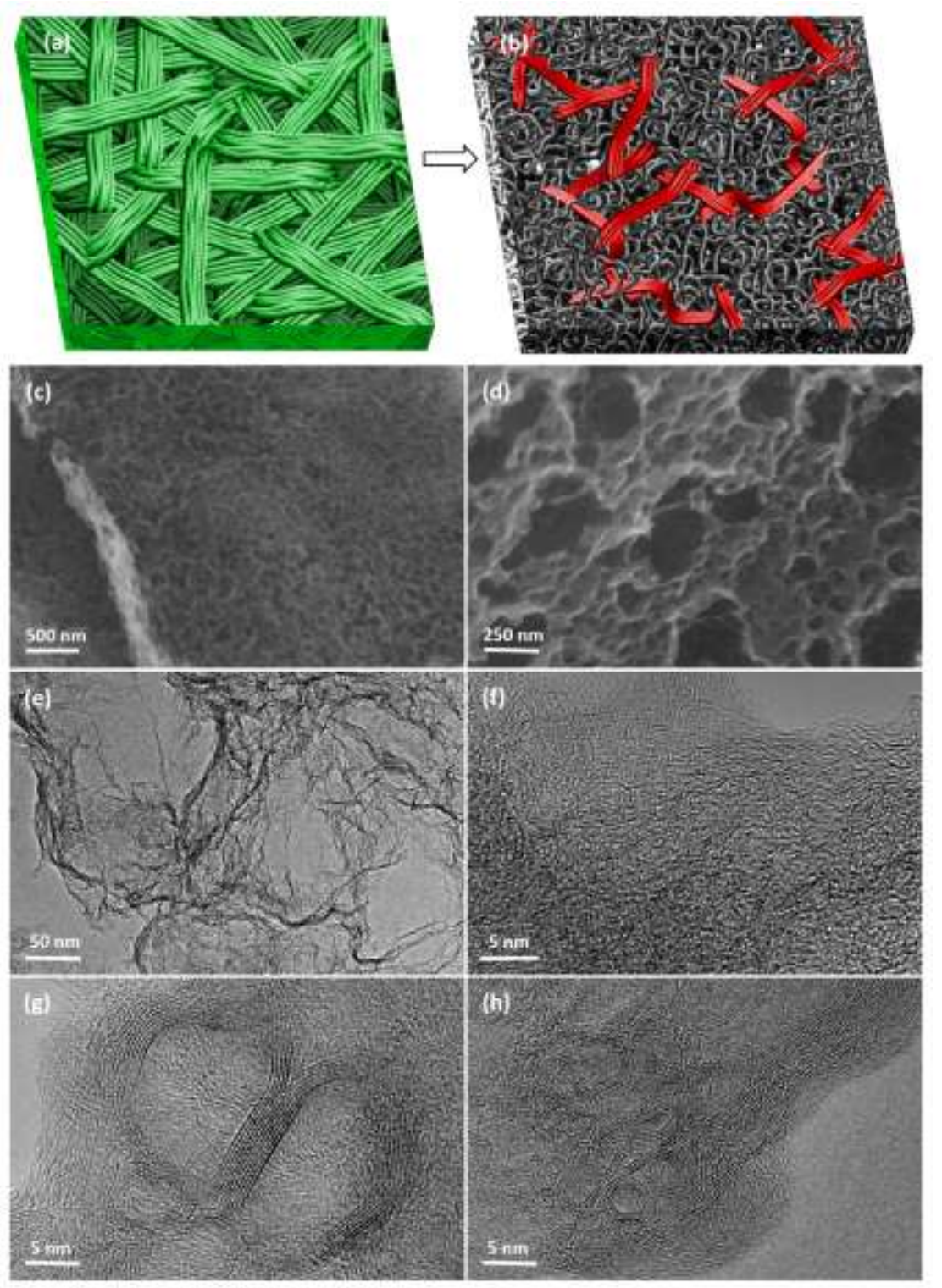

Figure 3 Schematic to illustrate the transformation of (a) CNC to (b) mesoporous carbon with a short-range ordered carbon lattice (black area) and percolated carbon nanofibers (red fiber). Morphology of carbonized CNC: SEM showing (c) the porous structure and (d) the carbon nanofiber wrapped hybrid structure; (e) TEM image showing the carbon nanosheet; HRTEM images showing (f) the hard carbon co-existing with the short-range ordered carbon, (g) carbon nanofiber with the graphitic crystalline pattern and $(\mathrm{h})$ the curved long range ordered carbon observed side-on. 
Figure $4 \mathrm{~b}$ is the HRTEM image of the $\mathrm{CNC}$ derived carbon from the area contained in the redsquared site in Figure 4a. Higher magnification HRTEM image of the area contained in the redsquared site in Figure $4 \mathrm{~b}$ is given in Figure $4 \mathrm{c}$ with the inset Fourier transform images showing the crystalline carbon structure. More HRTEM images are given in Figure S4 to support the crystalline carbon structure. The experimental data proves the formation of graphitic carbon from $\mathrm{CNC}$ under $1000{ }^{\circ} \mathrm{C}$ carbonization. We then used molecular dynamics (MD) simulation to explain the possible mechanism of ordered carbon formation from $\mathrm{CNC}$ nanorods. Our MD model is shown in Figure 4d, in which a cellulose bundle contains 36 cellulose chains (each with 4 repeat units) that are packed in a pattern as in a cellulose crystallized region. Figure $4 \mathrm{e}$ to $4 \mathrm{~h}$ show the decomposition process as the temperature increases. Details of simulation can be found in supporting information. At $600 \mathrm{~K}$, the cellulose bundle remains in a rather crystalline configuration. At $800 \mathrm{~K}$, this crystal shows signs of destabilization, as certain cellulose chains are dislocated from their original position. Further increase of temperature causes the gradual decomposition of the bundle. At $900 \mathrm{~K}$, a major portion of the original bundle has been depolymerization into various types of segments. We observe molecules of $\mathrm{H}_{2}, \mathrm{H}_{2} \mathrm{O}, \mathrm{CO}, \mathrm{CO}_{2}$, as well as carbon radicals. At $1300 \mathrm{~K}$, the entire cellulose bundle has been decomposed and more carbon radicals emerge. The zoomed-out image in Figure $4 \mathrm{~h}$ shows four carbon radicals and some other segments, such as $\mathrm{CO}, \mathrm{CO}_{2},-\mathrm{CH}_{2}$.

The effect of temperature is three-fold. Firstly, it enables the generation of enough carbon radicals, which increases the spatial density of carbon radicals. It also renders adequate kinetic energy to those carbon radicals. To form a graphene quantum dot, three conditions have to be satisfied. The spatial density of carbon radicals should allow a favorable possibility that there are 
enough pairs of carbon radicals that can potentially engage in collision. Secondly, the carbon radicals should possess enough kinetic energy, so that when they collide (best scenario: head-on; worst scenario: tailgate), there are enough carbon radicals to have a favorable possibility to overcome the van der Waals and electrostatic repulsive energy barrier and thus come close enough to form carbon-carbon bonds, as in the $\mathrm{C} 2$ pairs shown in Figure 4i. Thirdly, the spatial density of carbon radicals should continuously increase, allowing for the formation of graphenelike network patterns from the collision and coalescence of scattered, already-bonded carbon radicals. Figure $4 \mathrm{i}$ to $4 \mathrm{j}$ show the effect of continuously increased spatial density of carbon radicals, as seen in our MD simulations. Figure 4k shows the formation of many long carbon atom chains, by merging many $\mathrm{C} 2$ pairs and carbon radicals. Figure $4 \mathrm{j}$ show that a nucleation of a carbon dot occurs as the spatial density further increases, while the remaining amorphous region is composed of densely packed carbon chains. Such amorphous regions eventually transform into a graphene structure, as in Figure 41. In-situ TEM study (detailed in Figure S5) further proves the transformation of cellulose to crystalline carbon (Figure 4m) after carbonization at $1000{ }^{\circ} \mathrm{C}$. 


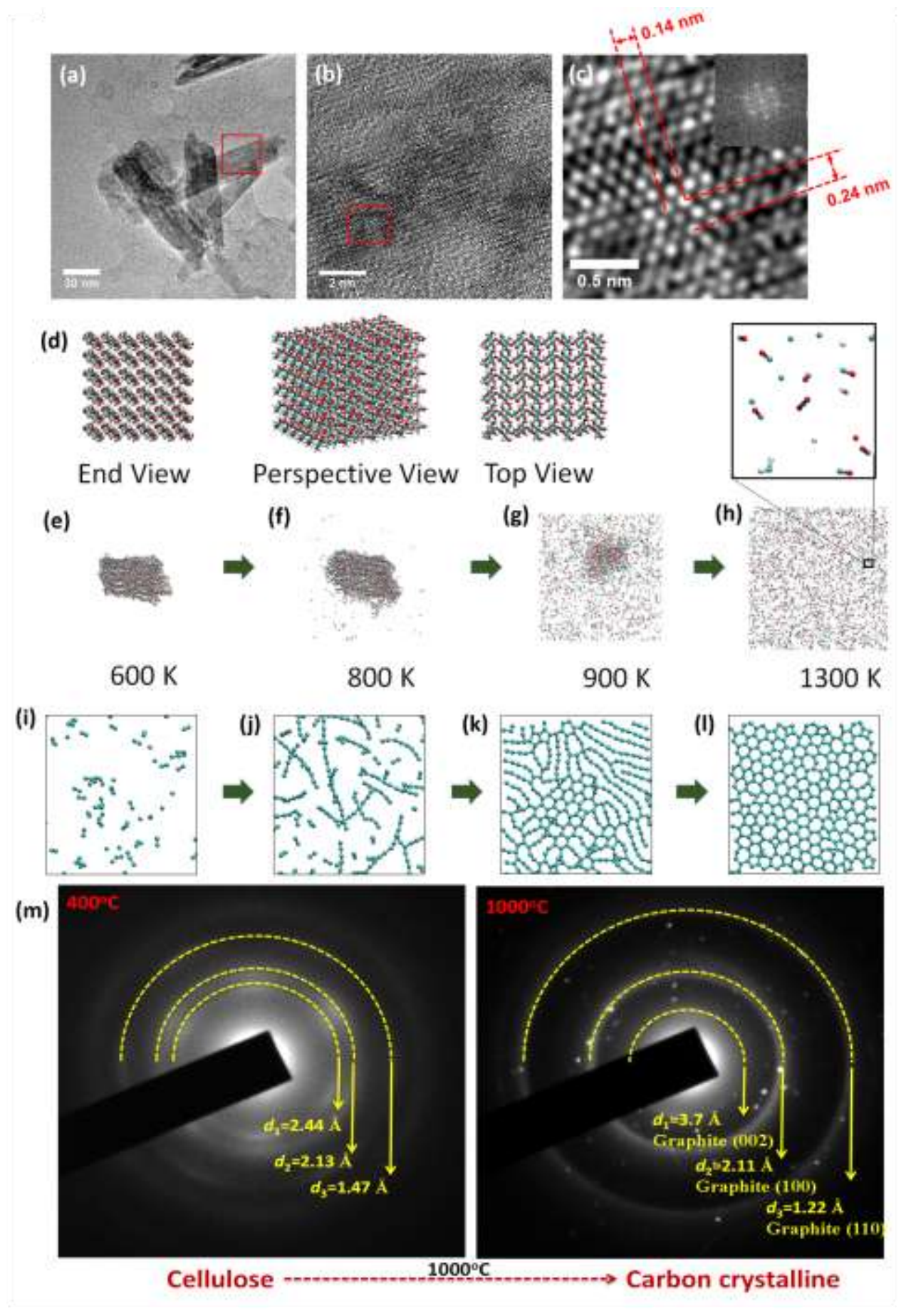

Figure 4 (a) TEM image of the CNC derived carbon (front-on); (b) Higher magnification HRTEM image of the area contained in the red squared in (a); (c) Higher magnification HRTEM image of the area contained in the red squared in (b) with the Fourier transform shown in the inset; (d) molecular dynamics model showing cellulose bundle containing 36 cellulose chains. 
Color code: cyan for carbon, red for oxygen, white for hydrogen. (e) to (h) show representative snapshots of the cellulose bundle during the increase of temperature. Each temperature is maintained for 100 ps. (i) to (1) show the formation of a graphene-like structure as the spatial density of carbon radicals increases. (m) In-situ TEM diffraction patterns at $400{ }^{\circ} \mathrm{C}$ and $1000{ }^{\circ} \mathrm{C}$ to show the transformation from cellulose to crystalline carbon.

We further characterized the properties of the obtained mesoporous carbon using nitrogen adsorption-desorption, X-ray photoelectron spectrum (XPS), Raman scattering and XRD. The specific Brunauer-Emmett-Teller (BET) surface areas of the mesoporous carbon is $145.56 \mathrm{~m}^{2} / \mathrm{g}$, and the Barrett-Joyner-Halenda (BJH) desorption cumulative pore volume between $1.7 \mathrm{~nm}$ to $30.0 \mathrm{~nm}$ is $0.06 \mathrm{~cm}^{3} / \mathrm{g}$. From the pore size distribution curve in Figure S6a, we conclude that most of the pores are distributed in the range of less than $3 \mathrm{~nm}$. The inset isothermal curve shows a typical type IV with a hysteresis loop characteristic of mesoporous structures. The XPS survey presented in Figure S6b has a broad, asymmetrical tail toward high binding energy, indicating a high concentration of $\mathrm{SP}^{2}$ carbon existing in the carbonized CNC. The XPS result is in good agreement with the HRTEM analysis. The Raman spectrum exhibits that G-band centered at $1575 \mathrm{~cm}^{-1}$ arising from the in-plane vibrations of the $\mathrm{sp}^{2}$-bonded crystallite carbon and has been observed for single crystal graphite. For polycrystalline graphite, however, another peak denoted as the 'disorder' peak (D-band) centered at $1350 \mathrm{~cm}^{-1}$ is typically observed, which is attributed to in-plane vibrations of $\mathrm{sp}^{2}$ bonded carbon within structural defects. The intensity ratio of D-band and G-band $\left(\mathrm{I}_{\mathrm{D}} / \mathrm{I}_{\mathrm{G}}\right)$ of carbonized CNC is 1.07 , as shown in Figure S6c. The XRD pattern in Figure S6d shows a typical hard carbon structure with two broad peaks at $2 \theta=23^{\circ}$ and $2 \theta=43^{\circ}$, which correspond to the interlayer scattering of graphene stacks. ${ }^{41}$ 
The low cost mesoporous carbon derived from $\mathrm{CNC}$ at a low carbonization temperature is ideal for high-performance SIBs for the following reasons. (1) The inter-layer distance is around 0.39 $\mathrm{nm}$ based on XRD studies, larger than the minimum distance required for the $\mathrm{Na}$ ion intercalation; ${ }^{42}(2)$ The small pore volume of 1.7- $30 \mathrm{~nm}$ based on BJH study is excellent for adsorbing enough electrolyte to support fast electrochemical reaction at high rate; (3) The crystalline ordered, carbon-nanofiber-structure (from TEM study) embedded within porous network (from SEM study) can provide fast electronic and ion transport for high-rate performance.

To evaluate the $\mathrm{Na}$ ion storage properties of the carbonized $\mathrm{CNC}$, coin cells are assembled using the free-standing carbonized $\mathrm{CNC}$ film as binder and current-collector free working electrodes (see details in experimental part). Galvanostatic charge/discharge cycling is performed in a potential window of $0.01-2.5 \mathrm{~V}$ versus $\mathrm{Na} / \mathrm{Na}^{+}$. Figure $5 \mathrm{a}$ shows the potential profiles of the carbonized $\mathrm{CNC}$ at different current densities. Interestingly, in contrast to typical hard carbon anode, we do not observe a long potential plateau near $0.0 \mathrm{~V}$ versus $\mathrm{Na} / \mathrm{Na}^{+}$in the potential profile of carbonized CNC. ${ }^{43}$ Moreover, two slopes are found; the first one between $2.5 \mathrm{~V}$ to 0.25 $\mathrm{V}$ is related to the storage of $\mathrm{Na}$ ions at defects or pores of the carbonized CNC. The later short slope below $0.25 \mathrm{~V}$ corresponds to the intercalation of $\mathrm{Na}$ ions in the ordered layers $(0.39 \mathrm{~nm}){ }^{44}$

The mesoporous carbon with a network structure also shows excellent kinetics and rate performance, which delivers $375 \mathrm{mAh} / \mathrm{g}, 350 \mathrm{mAh} / \mathrm{g}, 320 \mathrm{mAh} / \mathrm{g}$ and $220 \mathrm{mAh} / \mathrm{g}$ at $20 \mathrm{~mA} / \mathrm{g}, 40$ $\mathrm{mA} / \mathrm{g}, 100 \mathrm{~mA} / \mathrm{g}$ and $200 \mathrm{~mA} / \mathrm{g}$, respectively (Figure 5b). As the applied current density increases, the specific capacity slightly drops accordingly (Figure 5a). But no significant over- 
potential is observed, suggesting great electrochemical kinetics of the carbonized CNC electrode from the excellent electronic conductivity of the carbonized CNC. Long-term cycling is further performed at $100 \mathrm{~mA} / \mathrm{g}$ after a pre-cycling at $10 \mathrm{~mA} / \mathrm{g}$ for 5 cycles, as shown in Figure $5 \mathrm{c}$. Carbonized CNC electrode exhibits a stable capacity around $300 \mathrm{mAh} / \mathrm{g}$ with nearly $100 \% \mathrm{CE}$ over 400 cycles, suggesting a promising candidate for SIB anode. Furthermore, the stable specific capacity of $300 \mathrm{mAh} / \mathrm{g}$ at $100 \mathrm{~mA} / \mathrm{g}$ is one of the highest among the biomass derived carbon anodes for SIBs. ${ }^{45,46,47,48}$ In a control experiment, we also explored the Na ion storage properties of carbon derived from regular cellulose microfiber at $1000^{\circ} \mathrm{C}$. The HRTEM image of the carbonized regular cellulose microfiber is presented in Figure S7. There is no nano-sized graphitic domain observed. The cycling performance of regular cellulose microfiber derived carbon, as displayed in Figure S8, shows a much lower capacity than that of carbonized CNC (200 mAh/g vs $300 \mathrm{mAh} / \mathrm{g}$ at $100 \mathrm{~mA} / \mathrm{g}$ ). Moreover, rate capability of regular cellulose microfiber derived carbon (Figure S9) is also poor, due to low reaction kinetics. Note that the first cycle $\mathrm{CE}$ of carbonized $\mathrm{CNC}$ is relatively low at this stage (Figure S10), which could be further improved by using better electrolytes and modifying the surface. ${ }^{49}$ 

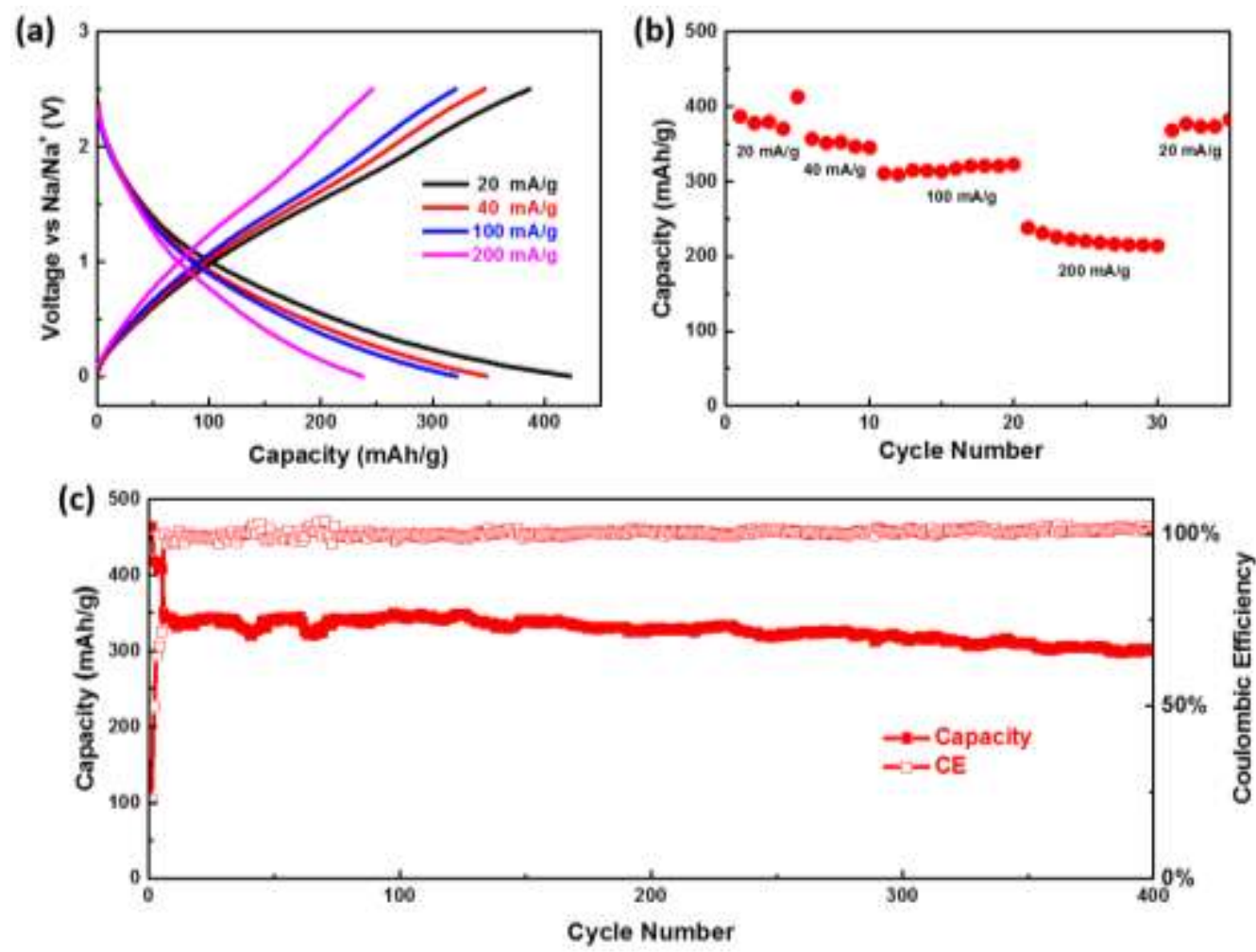

Figure 5 Electrochemical performances of carbonized CNC: (a) galvanostatic charge/discharge profiles at different current densities in a potential window of $0.01-2.5 \mathrm{~V}$ versus $\mathrm{Na} / \mathrm{Na}^{+}$; (b) rate performance at various current densities; and (c) cycling performance at $10 \mathrm{~mA} / \mathrm{g}$ for the first five cycles and then $100 \mathrm{~mA} / \mathrm{g}$, corresponding Coulombic efficiency (CE) in the right $\mathrm{Y}$ axis.

\section{Conclusion}

A unique mesoporous and percolated carbon was achieved by directly carbonizing $\mathrm{CNC}$ derived from trees. Crystalline ordered carbon was obtained at a relatively low carbonization temperature. The mesoporous carbon immersed with short-range ordered carbon lattice and percolated carbon nanofibers has the ideal structure and conductivity for SIB anode with outstanding performance including high capacity, excellent rate, and good cycling stability. A high starting capacity of $340 \mathrm{mAh} / \mathrm{g}$ at a high current density of $100 \mathrm{~mA} / \mathrm{g}$, maintaining $88.2 \%$ capacity over 400 cycles is 
achieved. Both TEM and molecular simulation support the formation of ordered hexagonal carbon rings from aligned cellulose glucose chains at a low temperature of $1000{ }^{\circ} \mathrm{C}$. The study not only provides a high performance hard carbon anode for sodium ion batteries, but also shed light on how to convert biomass to ordered conductive carbon at low temperatures.

\section{Acknowledgement:}

This work was also supported as part of the Nanostructures for Electrical Energy Storage (NEES), an Energy Frontier Research Center funded by the U.S. Department of Energy, Office of Science, Basic Energy Sciences under Award number DESC0001160. We thank the support of the Maryland Nanocenter and its Fablab and Nisplab. 


\section{References:}

1. $\quad$ E. Frackowiak, F. Béguin, Carbon 39 (2001) 937-950.

2. K. Keren, R. S. Berman, E. Buchstab, U. Sivan, E. Braun, Science 302 (2003) 1380-1382.

3. P. Avouris, J. Appenzeller, R. Martel, S. J. Wind, P. IEEE 91 (2003) 1772-1784.

4. J. Hass, R. Feng, T. Li, X. Li, Z. Zong, W. De Heer, P. First, E. Conrad, C. Jeffrey, C. Berger, Appl. Phys. Lett. 89 (2006) 143106-143109.

5. T. Palacios, Nature Nanotech. 6 (2011) 464-465.

6. J. Jiang, J. Zhu, W. Ai, Z. Fan, X. Shen, C. Zou, J. Liu, H. Zhang, T. Yu, Energy Environ. Sci. 7 (2014) 2670-2679.

7. X. Xie, W. T. Zhao, H. R. Lee, C. Liu, M. Ye, W. J. Xie, B. X. Cui, C. S. Criddle, Y. Cui, Acs Nano 8 (2014) 11958-11965.

8. G. Y. Zheng, S. W. Lee, Z. Liang, H. W. Lee, K. Yan, H. B. Yao, H. T. Wang, W. Y. Li, S. Chu, Y. Cui, Nature Nanotech. 9 (2014) 618-623.

9. H. L. Pan, Y. S. Hu, L. Q. Chen, Energy Environ. Sci. 6 (2013) 2338-2360.

10. S. Komaba, W. Murata, T. Ishikawa, N. Yabuuchi, T. Ozeki, T. Nakayama, A. Ogata, K. Gotoh, K. Fujiwara, Adv. Funct. Mater. 21 (2011) 3859-3867.

11. M. Dahbi, N. Yabuuchi, K. Kubota, K. Tokiwa, S. Komaba, Phys. Chem. Chem. Phys. 16 (2014) 15007-15028.

12. C. Bommier, X. L. Ji, Isr. J. Chem. 55 (2015) 486-507.

13. B. L. Ellis, L. F. Nazar, Curr. Opin. Solid State Mater. Sci. 16 (2012) 168-177.

14. H. Wang, Z. Li, J. K. Tak, C. M. B. Holt, X. Tan, Z. Xu, B. S. Amirkhiz, D. Harfield, A. Anyia, T. Stephenson, D. Mitlin, Carbon 57 (2013) 317-328.

15. H. Wang, Z. Xu, A. Kohandehghan, Z. Li, K. Cui, X. Tan, T. J. Stephenson, C. K. King'ondu, C. M. B. Holt, B. C. Olsen, J. K. Tak, D. Harfield, A. O. Anyia, D. Mitlin, Acs Nano 7 (2013) 5131-5141.

16. W. Chen, R. B. Rakhi, H. N. Alshareef, J. Mater. Chem. 22 (2012) 14394-14402.

17. E. M. Lotfabad, J. Ding, K. Cui, A. Kohandehghan, W. P. Kalisvaart, M. Hazelton, D. Mitlin, Acs Nano 8 (2014) 7115-7129.

18. W. Luo, B. Wang, C. G. Heron, M. J. Allen, J. Morre, C. S. Maier, W. F. Stickle, X. Ji, Nano Lett. 14(2014) 2225-2229.

19. F. Shen, H. Zhu, W. Luo, J. Wan, L. Zhou, J. Dai, B. Zhao, X. Han, K. Fu, L. Hu, ACS Appl. Mater. Interfaces 7 (2015) 23291-23296.

20. W. Luo, J. Schardt, C. Bommier, B. Wang, J. Razink, J. Simonsen, X. Ji, J. Mater. Chem. A 1 (2013) 10662-10666.

21. D. Bondeson, A. Mathew, K. Oksman, Cellulose 13 (2006) 171-180.

22. D. Klemm, B. Heublein, H. P. Fink, A. Bohn, Angew. Chem. Int. Ed. 44 (2005) 33583393.

23. J. P. F. Lagerwall, C. Schutz, M. Salajkova, J. Noh, J. Hyun Park, G. Scalia, L. Bergstrom, NPG Asia Mater. 6 (2014) e80.

24. Y. Habibi, L. A. Lucia, O. J. Rojas, Chem. Rev. 110 (2010) 3479-3500.

25. F. Dri, L. Hector, Jr., R. Moon, P. Zavattieri, Cellulose 20 (2013) 2703-2718.

26. R. J. Moon, A. Martini, J. Nairn, J. Simonsen, J. Youngblood, Chem. Soc. Rev. 40 (2011) 3941-3994.

27. G. Nystrom, A. B. Fall, L. Carlsson, L. Wagberg, Cellulose 21 (2014) 1591-1599.

28. A. G. Dumanli, A. H. Windle, J. Mater. Sci. 47 (2012) 4236-4250. 
29. W. E. Tenhaeff, O. Rios, K. More, M. A. McGuire, Adv. Funct. Mater. 24 (2014) 86-94.

30. G. S. Oleynik, L. N. Pereselentseva, L. R. Vishnyakov, E. L. Vishnyakova, A. V. Kotko, Tech. Phys. Lett. 37 (2011) 233-236.

31. C. K. Chan, H. L. Peng, G. Liu, K. McIlwrath, X. F. Zhang, R. A. Huggins, Y. Cui, Nature Nanotech. 3 (2008) 31-35.

32. J. C. Meyer, C. Kisielowski, R. Erni, M. D. Rossell, M. F. Crommie, A. Zettl, Nano Lett. 8 (2008) 3582-3586.

33. M. Pozzo, D. Alfè, P. Lacovig, P. Hofmann, S. Lizzit, A. Baraldi, Phys. Rev. Lett. 106 (2011) 135501.

34. Y. M. Wang, M. W. Chen, F. H. Zhou, E. Ma, Nature 419 (2002) 912-915.

35. M. M. de Souza Lima, R. Borsali, Macromol. Rapid Commun. 25 (2004) 771-787.

36. Y. Habibi, L. A. Lucia, O. J. Rojas, Chem. Rev. 110 (2010) 3479-3500.

37. D. Klemm, F. Kramer, S. Moritz, T. Lindstrom, M. Ankerfors, D. Gray, A. Dorris, Angew. Chem. Int. Ed. 50 (2011) 5438-5466.

38. R. J. Moon, A. Martini, J. Nairn, J. Simonsen, J. Youngblood, Chem. Soc. Rev. 40 (2011) 3941-3994.

39. M. Giese, L. K. Blusch, M. K. Khan, M. J. MacLachlan, Angew. Chem. Int. Ed. 54 (2015) 2888-2910.

40. A. Kumar, Y. S. Negi, V. Choudhary, N. K. Bhardwaj, J. Mater. Phys. Chem. 2 (2014) 18.

41. B. Jache, C. Neumann, J. Becker, B. M. Smarsly, P. Adelhelm, J. Mater. Chem. 22 (2012) 10787-10794.

42. Y. Cao, L. Xiao, M. L. Sushko, W. Wang, B. Schwenzer, J. Xiao, Z. Nie, L. V. Saraf, Z. Yang, J. Liu, Nano Lett. 12 (2012) 3783-3787.

43. D. A. Stevens, J. R. Dahn, J. Electrochem. Soc. 147 (2000) 1271-1273.

44. D. A. Stevens, J. R. Dahn, J. Electrochem. Soc. 148 (2001) A803-A811.

45. Y. Yan, Y. X. Yin, Y. G. Guo, L. J. Wan, Adv. Energy Mater. 4 (2014) 1079-1098.

46. S. Komaba, W. Murata, T. Ishikawa, N. Yabuuchi, T. Ozeki, T. Nakayama, A. Ogata, K. Gotoh, K. Fujiwara, Adv. Funct. Mater. 21 (2011) 3859-3867.

47. K.-1. Hong, L. Qie, R. Zeng, Z.-q. Yi, W. Zhang, D. Wang, W. Yin, C. Wu, Q.-j. Fan, W.-x. Zhang, Y.-h. Huang, J. Mater. Chem. A 2 (2014) 12733-12738.

48. X. Zhou, Y.-G. Guo, ChemElectroChem 1 (2014) 83-86.

49. Y. Wen, K. He, Y. Zhu, F. Han, Y. Xu, I. Matsuda, Y. Ishii, J. Cumings, C. Wang, Nat. Commun. 5 (2014) 4033-4042. 
Graphical Abstract
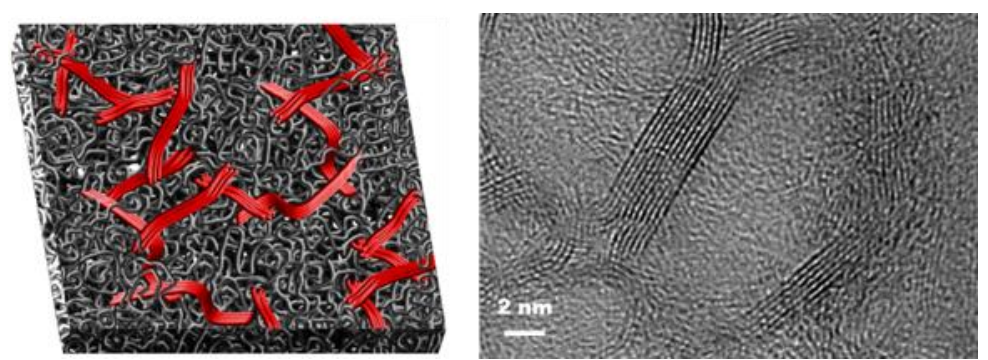

We demonstrated that from ordered cellulose nanocrystals, high quality mesoporous carbon with an increased short-range ordered lattice and a high specific ratio of percolated graphitic nanofibers can be obtained, even at a relatively low carbonization temperature. The mesoporous carbon exhibits excellent kinetics and rate performance when used as sodium-ion battery anode. 